\title{
A 3D-FEM-Based Model to Predict the Electrical Constriction Resistance of Compressed Contacts
} Jordi-Roger Riba ${ }^{1}$, Antonio-Giuseppe Mancini ${ }^{2}$, Carlos Abomailek ${ }^{1}$ and Francesca Capelli ${ }^{1}$

${ }^{1}$ Universitat Politècnica de Catalunya, 08222 Terrassa (Barcelona), Spain

${ }^{2}$ Università degli Studi del Sannio, 82100 Benevento, Italy

\begin{abstract}
This work proposes a method to estimate the electrical constriction resistance of two mating metallic rough surfaces based on the finite element method (FEM). The FEM-based method generates a random array of three-dimensional orthogonal parallelepipeds to simulate the stochastic distribution of the asperities across the contact interface. The effect of the contact pressure is studied in detail, since once the contact materials and the topology of the contact area are settled, the contact pressure plays a critical role in determining the electrical constriction resistance. The proposed model is based on two critical variables, the contact pressure and the surface roughness of the mating surfaces, which must be measured in the laboratory to calibrate the model. Results provided by the FEM-based model are compared with experiments for three geometries, thus validating the accuracy of the proposed approach. Although the apparent contact areas of the analyzed specimens have a rectangular shape, the proposed method is also applicable to determine the electrical constriction resistance of other geometries. It is also proved that depending on the pressure applied to the contact interface, the electrical constriction resistance can be almost independent of the apparent area of contact. Although the aim of this work was to generate an electrical constriction resistance model for power connectors, it is also applicable to many other power devices.
\end{abstract}

Keywords - Finite element method, constriction resistance, conducting contact area, apparent contact area.

\section{INTRODUCTION}

The contact resistance greatly determines the energy-efficiency, thermal behavior, stable operation and long term service of electrical connections [1]. To obtain an improved electrical connection, the electrical contact resistance must be low and stable over time, otherwise it can cause overheating and a reduction of the service life of the associated electrical device.

The contact resistance of a joint depends on the apparent or nominal contact area [2] as well as the morphology and distribution of the conducting spots across the contact interface [3]. It is well known that the surface condition and the mechanical pressure applied have a deep impact on the real area of contact and the distribution and density of conducting spots. The highest asperities of the mating surfaces come into contact, forming the real contact spots defining the real contact area. However, the spots are multiply connected instead of continuous, since they are formed by a set of subspots (nanometric scale) generating 
the physical area of contact which coincide with the conducting contact area of current conductivity that plays a key role for calculating the electrical contact resistance [4]. When two rough surfaces are in contact each other, the apparent contact area (geometrical or nominal area) $A_{a}$ is much greater than the real contact area $A_{r}$. The real area of contact, $A_{r}$, usually embraces the load-bearing area, the latter one often containing oxide films. The conducting contact area, where there is the strong electrical interaction between the two surface in contact, is only a small fraction $(<<1 \%)$ of $A_{r}[4]$. For simplicity, the spots are often supposed to be circular with radius $a$ covering the conducting contact area [5].

It is a recognized fact that bolted connections allow performing compact and reliable aluminum and copper contacts [6] although it can lead to irregular contact pressure.-When increasing the mechanical load applied to the contacting surfaces, the area of contact tends to increase [7] and thus the number and the area of spots. These spots provide the only conducting pathways for the transfer of electrical current between the two contacting rough surfaces [6].

The contact pressure must be enough to ensure a sufficient area of contact, thus permitting a continuous transfer of electrical current across the interfaces in contact. In [8] it is proved that the contact pressure has a deep impact on contact temperature, since an increasing contact pressure tends to reduce the temperature in the contact area. The asperities limit the real contact area between the mating surfaces, which depends on the topology of such surfaces, material properties and contact pressure [9]. To describe the contact between two rough surfaces, both the topography of the mating surfaces and the mechanical deformation of the asperities must be considered [10].

Because of the spots, the current lines tend to narrow in the proximities of the spots. This constriction effect increases the effective electrical resistance since the current lines are unevenly distributed across the cross section of the nominal contact area, thus increasing the effective resistance, which is known as constriction resistance. The constriction resistance goes beyond the microscale effect, thus playing an important role due to the mismatch between the cross sections of the spots and the nominal area of contact [9]. The effective resistance also increases due to the formation of oxide and contaminating films in the joint interface. The contact resistance takes into account both components, that is, the constriction term and the effect of the contaminant films [4]. Elastic and plastic deformation of the interface structures has a deep impact on the constriction resistance [9], which greatly influences the reliability, performance and service life of electrical contacts [11] and thus of power connectors, relays and other electrical devices [12].

Surface roughness is often categorized into nanoscale roughness or subroughness, roughness, waviness and errors in form. These roughness levels are associated to the different areas of contact, that is, physical, real and apparent [4]. It is a recognized fact that surface roughness presents a multiscale nature, that is, the size of asperities can range from atomic scale to sample length. Surface asperities exhibit a stochastic distribution 
of the characteristic parameters such as height, or slope [4].

There are different approaches to describe the contact of rough surfaces, the most applied based on statistical, fractal and multiscale approaches [7]. Statistical models are based on the stochastic nature of contact surfaces, the model of Greenwood and Williamson [13] being the most widely applied. Statistical approaches often require few parameters, so they are simple and fast. However, the selection of model parameters can be conflicting [14] because of the scale ambiguity, since the value of the average curvature radius of the surface profile depends on the scale of observation [10]. Another possibility is to apply a fractal approach to reproduce the rough contact surfaces. Fractal models account for the multiscale nature of the contact surfaces, which is not considered by the statistical approaches [7]. Multiscale models were initially developed as an alternative to fractal approaches taking into account the elastic-plastic conditions in the contact area. The finite element method (FEM) has also been extensively applied to analyze the electrical, mechanical [14] and thermal [15] behavior of rough mating surfaces, even by applying a multiphysics approach [9], [12], [16]-[20]. FEM approaches also allow considering both steady-state and transient conditions in the contact interface [11], [21]. However, most of the abovementioned works based on FEM analyses are not focused to develop a model of the electrical constriction resistance for rough surfaces with generalization capability as done in this work. Most of them are focused on analyzing specific geometries, except [9], which generates an array of small cylinders to simulate the asperities for building a model of the thermal constriction resistance.

Due to different contributing factors, it is challenging to determine a general formula to determine the contact resistance of solid interfaces [22]. This paper proposes a 3D-FEM approach to determine the electrical constriction resistance of two rough contacting surfaces as a function of the contact pressure when the surface roughness, geometry and properties of the contact materials are known. For the sake of simplicity, the 3DFEM model assumes orthogonal parallelepiped asperities randomly distributed across the apparent contact area. The density of asperities is supposed to be a function of the surface roughness, the apparent area of contact and the pressure applied to the contacts. The proposed model has generalization capability, since it

allows calculating the density of spots and the constriction resistance as a function of the pressure for a wide diversity of geometric configurations and materials. It can be useful during the design stage of several electrical devices with electrical contacts, such as power connectors or switches among others, since the contact resistance has a key role on their thermal performance and expected service life.

\section{THE CONTACT INTERFACE MODEL}

This section describes the FEM simulations conducted to predict the constriction resistance as a function of the ratio between the conducting and the apparent area of contact at the interface, $f=A_{c} / A_{a}$. It is assumed 
that the ratio $f$ depends on the contact pressure, topology and material properties of the contacts.

It is an accepted fact that the surface roughness forces in the contact occur within a finite number of spots [18], this number depending on the mechanical load applied to the contact. This phenomenon greatly determines the constriction resistance due to the constriction of the electrical current flowing through the asperities. The 3D-FEM approach applied in this paper is inspired in the model presented in [23], which assumes that the geometric resistance can be calculated from the geometry shown in Fig. 1 as,

$$
R_{\text {geom }}=\rho \frac{2 \cdot L}{A_{a}}+\rho \frac{d}{f \cdot A_{a}}
$$

$\rho$ being the electrical resistivity of the bulk material and that of the spots, $L$ the considered length of each contacting surface, $d$ the average height of the spots, $A_{a}$ the apparent or nominal contact area and $f=A_{c} / A_{a}$ the ratio between the conducting and the apparent contact areas at the interface, as shown in Fig. 1. It is noted that (1) can be easily obtained from pure geometric considerations from the geometry shown in Fig. 1b.

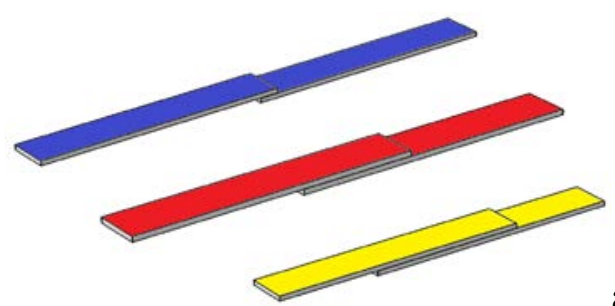

a)

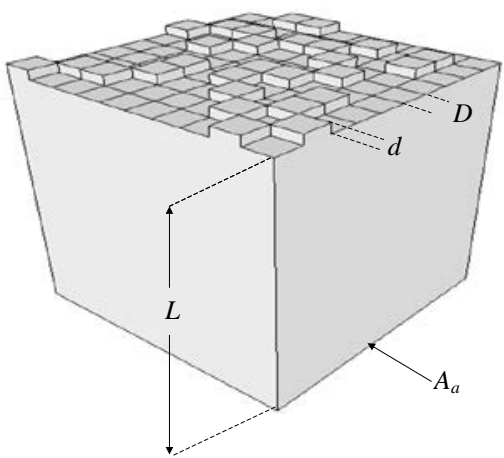

b)

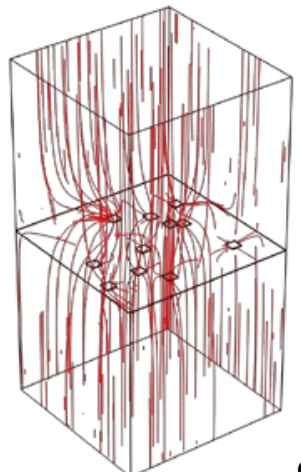

c)

Fig. 1. Copper specimens and geometry considered to determine the electrical constriction resistance. a) The three specimens considered with an apparent area of contact $A_{c}$ of dimensions $15 \times 32 \mathrm{~mm}^{2}, 60 \times 32 \mathrm{~mm}^{2}$ and $120 \times 32 \mathrm{~mm}^{2}$. b) The bottom contacting surface and the interface with the spots linking the two contacting surfaces. The upper contacting surface is considered as a flat surface with a thickness $L$ like the bottom one. c) Constriction of the current lines obtained through FEM simulations.

However, (1) is obtained from pure geometric considerations [23], since it only considers the increase of the electrical resistance due to the physical presence of spots but does not account for the constriction of the current lines, which reduces the effective cross sectional area, thus increasing the effective resistance. The 3D-FEM model presented in this paper overcomes the abovementioned drawbacks since it considers both, the geometrical resistance due to the spots and the constriction/spreading of the current lines due to the spots, as shown in Fig. 1c.

As displayed in Fig. 1, the proposed model of the contact interface formed by the two adjacent faces has a thickness $d$. It is subdivided in tinny squares or spots of dimensions $D x D$ and height $d$ which are randomly distributed within the apparent area of contact $A_{a}$ to cover a fraction $f=A_{c} / A_{a}$ where $A_{c}=n \cdot D \cdot D$, $n$ being the total number of spots and $A_{c}$ the total conducting area of the interface. To make the problem 
solvable, some simplifications must be applied. All the asperities are assumed to be identical with a rectangular cuboid shape (although a cylindrical geometry can be applied obtaining similar results, as described in Section 5) and randomly distributed across the contact area thus forming a random interface between the two contacting surfaces, as it is widely recognized [4]. The spots are supposed to be clean and to have the same electrical conductivity than the contacting surfaces since the spots and the contacting surfaces are made of the same material.

The 3D-FEM approach calculates the constriction resistance as the voltage drop between the two contacting surfaces divided by the current injected. The constriction resistance resulting from the 3D-FEM model, $R_{c, F E M}$, is calculated as the result of the 3D-FEM simulation, $R_{\text {simulated, }}$ minus the bulk resistance of the material, Rbulk, as

$$
R_{c, F E M}=R_{\text {simulated }}-R_{\text {bulk }}=R_{\text {simulated }}-\rho \cdot \frac{2 \cdot L}{A_{a}}
$$

The constriction resistance, $R_{C, F E M}$, has two terms, the first one owing to the physical constriction

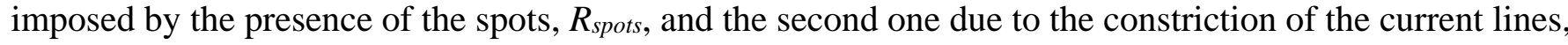
$R_{\text {current_lines, }}$ which is included in the results provided by the 3D-FEM simulation,

$$
R_{c, F E M}=R_{\text {spots }}+R_{\text {current_lines }}=\rho \cdot \frac{d}{f \cdot A_{a}}+R_{\text {current_lines }}
$$

In this work it is assumed that, for a given contact interface, $f$ depends on the pressure applied to the contact area, and thus $f=f(P)$. Fig. 2 shows the meshes of the geometries dealt with to calculate the constriction resistance through FEM simulations.
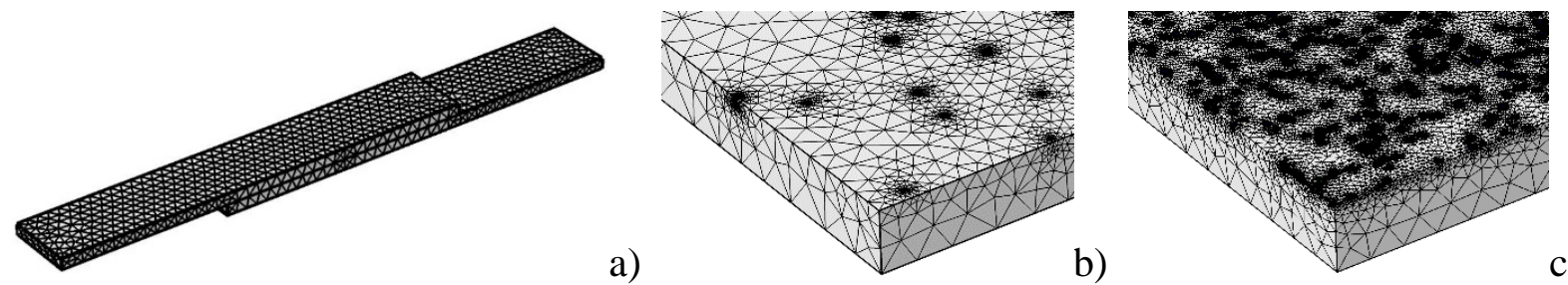

Fig. 2. FEM models. a) Three-dimensional tetrahedral mesh of the entire domain. b) Mesh with $f=0.00001$. c) Mesh with $f=$ 0.00004 .

The numerical values of the ratio $f$ between the conducting and the apparent area of contact must be very low, since the conducting contact area is far less than the apparent contact area, as described in Section 5. Therefore, the strong interaction between the two mating surfaces will occur within the conducting contact area.

It is noted that, although the 3D-FEM model does not directly considers the elastic-plastic behavior of the spots, this effect is already considered in the proposed approach since the number of spots modelled increases with the contact pressure because of the dependence $f=f(P)$. 


\section{THE PROPOSED APPROACH}

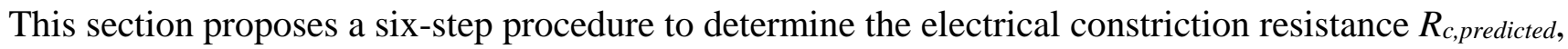
which is summarized in the flow chart shown in Fig. 3.

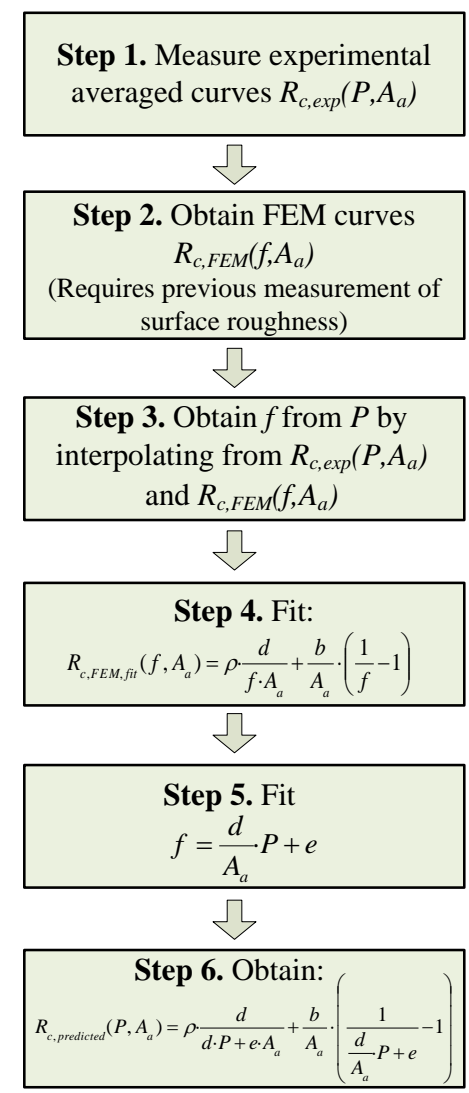

Fig. 3. Flowchart of the six-step procedure proposed in this paper.

The six-step procedure is detailed in the following paragraphs.

- Step 1. The dependence of the experimental constriction resistance with the contact pressure is determined. It is done using different samples with an apparent contact area $A_{a}$ of $15 \times 32 \mathrm{~mm}^{2}, 60 \times 32$ $\mathrm{mm}^{2}$ and $120 \times 32 \mathrm{~mm}^{2}$ each. Due to the statistical nature of $R_{c}$, 36 specimens were evaluated, 12 of each geometry, to obtain the averaged experimental curves $R_{c, \exp }\left(P, A_{a}\right)$ to deal with the great variability among copper samples.

- Step 2. Determine the FEM $R_{c, F E M}\left(f, A_{a}\right)$ curves as a function of $f$ and $A_{a}$. To this end, different samples with the same dimensions of those measured in Step 1 are simulated $\left(15 \times 32 \mathrm{~mm}^{2}, 60 \times 32 \mathrm{~mm}^{2}\right.$ and 120x32 $\mathrm{mm}^{2}$ ) within a suitable interval of the ratio $f$. To account for the stochastic nature of the constriction resistance, the identical rectangular cuboid spots were randomly distributed across the contact area.

- Step 3. In this stage the FEM generated curves $R_{c, F E M}\left(f, A_{a}\right)$ are related to the experimental curves $R_{c, \exp }\left(P, A_{a}\right)$ by interpolation. To this end, once fixed $A_{a}$, the pairs $(P, f)$ are obtained for each curve. 
- Step 4. For convenience, the curves $R_{c, F E M}\left(f, A_{a}\right)$ are fit to equation (4), which tries to reproduce the behavior of (3),

$$
R_{c, F E M, f i t}\left(f, A_{a}\right)=\frac{a}{f \cdot A_{a}}+\frac{b}{A_{a}} \cdot\left(\frac{1}{f}-1\right)=\rho \cdot \frac{d}{f \cdot A_{a}}+\frac{b}{A_{a}} \cdot\left(\frac{1}{f}-1\right)
$$

It is noted that the first term in (4) is due to the constrictive contribution because of the physical presence of the spots, whereas the second term is the resistance increase due to the constriction of the current lines. When $f=1$, that is, when there is no constrictive effect, (4) results in the classical expression $R_{c, F E M, f i t}\left(f, A_{a}\right)=\rho \cdot L_{\text {interface }} / A_{a} \cdot$ In the analyzed case, since the electrical resistivity of copper is $\rho_{\mathrm{Al}}=16.8$ $\mu \Omega \cdot \mathrm{mm}$ and $d=0.5 \mu \mathrm{m}=5 \cdot 10^{-4} \mathrm{~mm}$, (4) results in,

$$
R_{c, F E M, f i t}\left(f, A_{a}\right)=\frac{0.0084}{f \cdot A_{a}}+\frac{b}{A_{a}} \cdot\left(\frac{1}{f}-1\right)(\mu \Omega)
$$

where $A_{a}$ is expressed in $\mathrm{mm}^{2}$ and $f$ is dimensionless.

- Step 5. Once the pairs $(P, f)$ are known from Step 3, they are related by means of the following linear relationship,

$$
f=\frac{d}{A_{a}} \cdot P+e
$$

- Step 6. Finally, (6) is substituted into (4), so the curves $R_{c, p r e d i c t e d}\left(P, A_{a}\right)$ emerge. The expression of the $R_{c, p r e d i c t e d}\left(P, A_{a}\right)$ curves is as follows,

$$
R_{c, \text { predicted }}\left(P, A_{a}\right)=\rho \cdot \frac{d}{d \cdot P+e \cdot A_{a}}+\frac{b}{A_{a}} \cdot\left(\frac{1}{\frac{d}{A_{a}} \cdot P+e}-1\right)
$$

It is noted that (7) is the expression suggested in this paper to predict the contact resistance of a contact when the surface roughness, electrical resistivity of the contact materials, contact pressure and apparent contact area are known.

\section{MEASUREMENTS}

\section{A. Surface roughness measurement}

According to the international standard EN-ISO 4287 [24], the root-mean-square roughness $\mathfrak{R}_{q}[\mathrm{~m}]$ is calculated as,

$$
\mathfrak{R}_{q}=\sqrt{\frac{1}{n} \sum_{i=1}^{n} z_{i}^{2}}
$$


$n$ being the number of points considered within the sampling length $L$, and $z_{i}$ the roughness height value at the $i$-th point.

Surface roughness measurements of different copper samples were carried out by means of a Veeco Wyko 9800NT optical interferometer. It provides reconstructed images as that shown in Fig. 4.

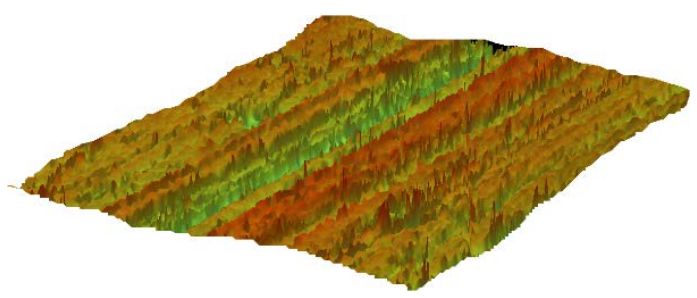

Fig. 4. Surface roughness reconstruction with the optical interferometer (95 $\mu \mathrm{m} \times 126 \mu \mathrm{m})$ of the analyzed copper samples.

Since for the copper samples dealt with the average value of 20 measurements of $\mathfrak{R}_{q}$ was approximately $0.25 \mu \mathrm{m}$, the total height of the spots (two mating interfaces) was considered as $d=0.5 \mu \mathrm{m}$ (see Fig. 1) in the FEM simulations. It is noted that although an optical interferometer was used to measure the $\mathfrak{R}_{q}$ surface roughness, this measure can also be performed with more affordable instruments such as stylus profilers.

\section{B. Pressure application and measurement}

The pressure was applied to the test samples and measured by means of a vertical $50 \mathrm{kN}$ computerized press testing machine BZ1-MM14780.ZW02 from Zwick Roell operating in compression mode. During the load tests, the electrical resistance of the copper specimens dealt with was measured simultaneously as described below.

\section{Resistance measurement}

To determine the resistance of the test samples, the 4-wire method was applied since the measures provided are independent of the resistance of the probes [25]. It requires two outer terminals for current injection and two inner terminals to measure the voltage. A Micro-Centurion II digital micro-ohmmeter from Raytech was used for this purpose, which allows injecting a dc current in the range 0-200 ADC and ensures an accuracy of $\pm 0.01 \mu \Omega \pm 0.1 \%$ reading. The constriction resistance was calculated as,

$$
R_{c, \text { measured }}=R_{\text {micro-ohmeter }}-R_{\text {bulk,FEM }}
$$

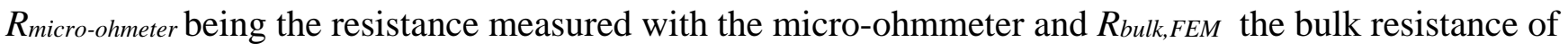
the material between the voltage terminal points of measurement obtained by means of FEM simulations when supposing a perfect contact, that is, neglecting any constrictive effect. Since the resistance is temperature dependent, all values were converted to a fixed reference temperature $\left(20^{\circ} \mathrm{C}\right)$, by applying the following correction,

$$
R_{20^{\circ} \mathrm{C}}=R(T) /[1+\alpha \cdot(T-20)]
$$


where the temperature coefficient of copper is $\alpha_{C u}=0.00391 /{ }^{\circ} \mathrm{C}$.

\section{RESULTS}

This section describes the results attained in this work when dealing with the copper samples shown in Fig. 5a. To obtain the experimental $R_{c, \exp }\left(P, A_{a}\right)$ curves both the vertical press machine and the microohmmeter described in Section 4 were used to simultaneously apply and measure the pressure applied to the apparent contact area and measure the electrical resistance as shown in Fig. 5b.

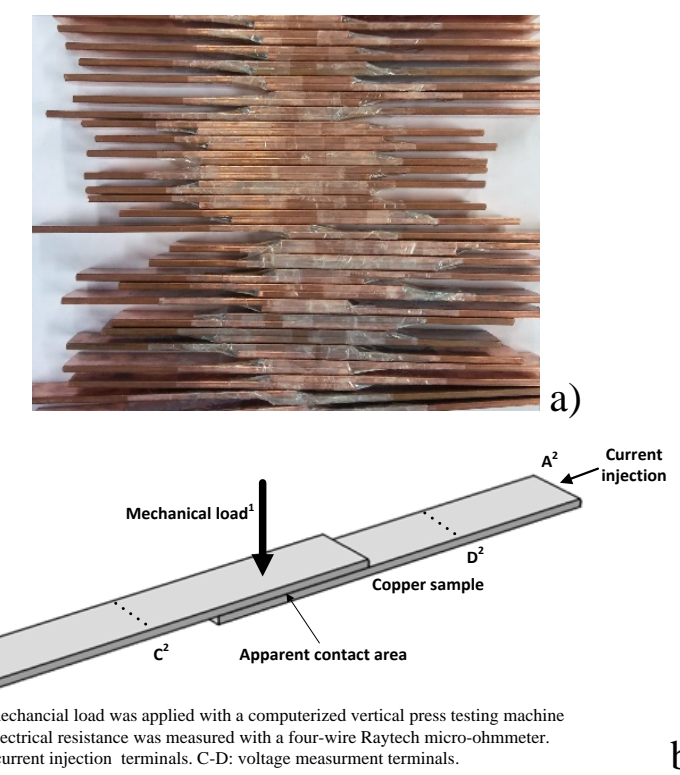

Fig. 5. a) Some of the analyzed copper samples dealt to obtain the experimental results presented in this paper. They include three sets of samples with $15 \times 32 \mathrm{~mm}^{2}, 60 \times 32 \mathrm{~mm}^{2}$ and $120 \times 32 \mathrm{~mm}^{2}$ apparent contact area $A_{a}$. b) Schematic of the tests applied.

When dealing with mechanical power connectors, the pressure exerted by the bolts-nuts elements is in the order 10-20 MPa. The reduced modulus of elasticity, which is defined in [26], is $E^{\prime}=65 \mathrm{GPa}$ for two mating copper surfaces, so it results in $P / E^{\prime} \sim 10^{-4}$. Results presented in [27] suggest that when dealing with samples with sub-micrometric surface roughness, and $P / E^{\prime} \sim 10^{-4}$ it results $f=A_{c} / A_{a}<10^{-4}$. Therefore, in Step 2 of the six-step procedure, the values of $f$ are chosen accordingly.

For each loading and partial unloading cycle, the curve is plotted between load with depth which provides information about hardness, elastic modulus and plasticity of the material under analysis.

Once measured the average surface roughness of the test samples, the height of the simulated spots is known $(d=0.5 \mu \mathrm{m})$, the dimensions of the square side of the spots considered in the 3D-FEM simulations can be determined from Fig. 6. According to Fig. 6, when $d=0.5 \mu \mathrm{m}$, the spacing of the spots is of about $D$ $=5 \mu \mathrm{m}$ (see Fig. 1). These values are applied to create the geometry for the 3D-FEM simulations. 


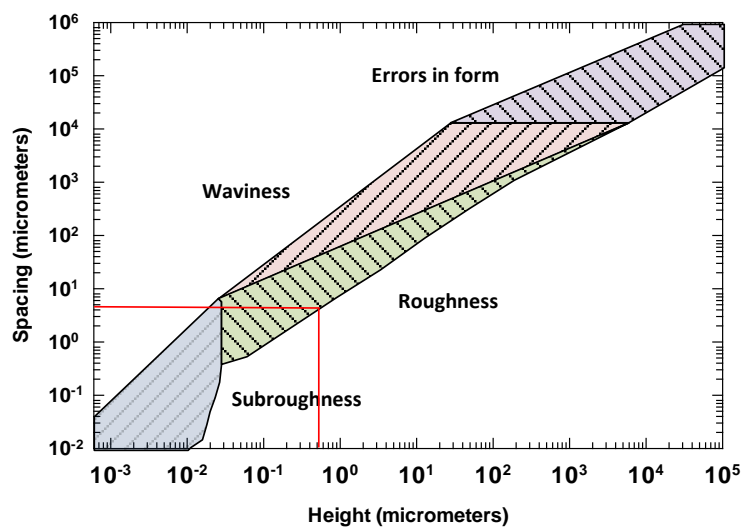

Fig. 6. Relation between the spots height and the spacing. Adapted from [4].

Table I summarizes the details of the contact spots considered in this work.

TABLE 1

DETAILS OF THE CONTACT SPOTS

\begin{tabular}{cc}
\hline \hline Shape of spots & Rectangular cuboid \\
Height of the spots & $d=0.5 \mu \mathrm{m}$ \\
Spacing between spots & $D=5 \mu \mathrm{m}$ \\
Spot area & $A_{\text {spot }}=D \times D=5 \mu \mathrm{m} \times 5 \mu \mathrm{m}=25 \mu \mathrm{m}^{2}$ \\
Position of spots & Random \\
Number of spots & $f \cdot A_{a} / A_{\text {spot }}$ \\
\hline \hline
\end{tabular}

All simulations performed in this paper are based on rectangular cuboid spots. However, a preliminary study showed that the shape of the spots has a little influence on the results of the contact resistance obtained through FEM simulations.

Next, the results of the six-step procedure are presented, step by step. Fig. 7a shows the three experimental curves $R_{c, \exp }\left(P, A_{a}\right)$ obtained with the tree samples sets dealt with (Step 1), that is with $A_{a}=$ $15 \times 32 \mathrm{~mm}^{2}, A_{a}=60 \times 32 \mathrm{~mm}^{2}$ and $A_{a}=120 \times 32 \mathrm{~mm}^{2}$, which were obtained as the average value of 12 repetitions each because of the great variability among the copper samples. The constriction resistance curves $R_{c, F E M}\left(f, A_{a}\right)$ obtained through FEM simulations (Step 2) are summarized in Fig. 7b.
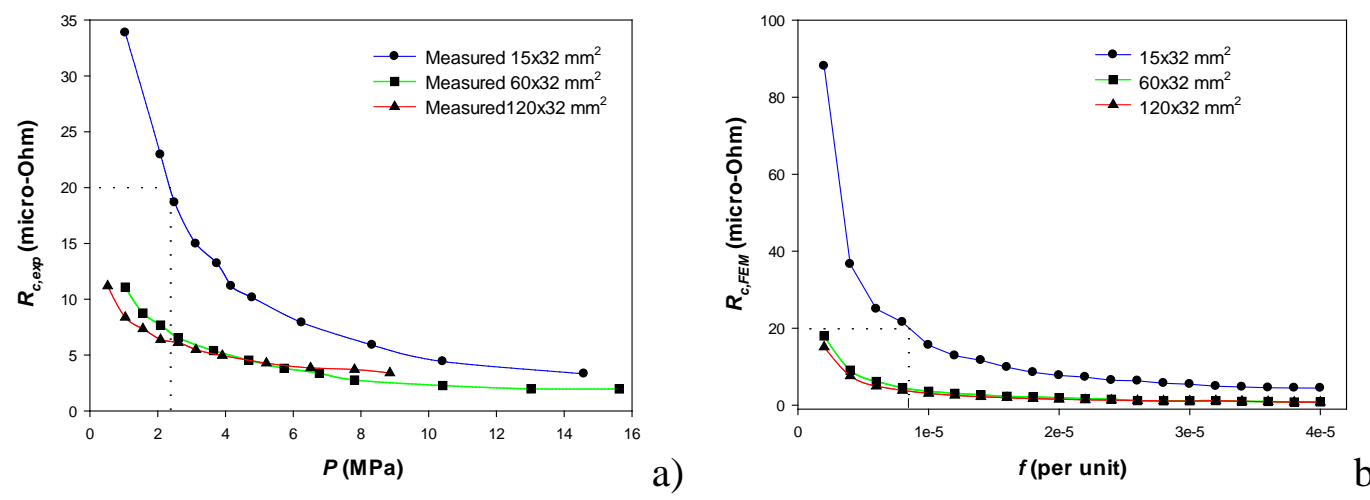

a)

b) 


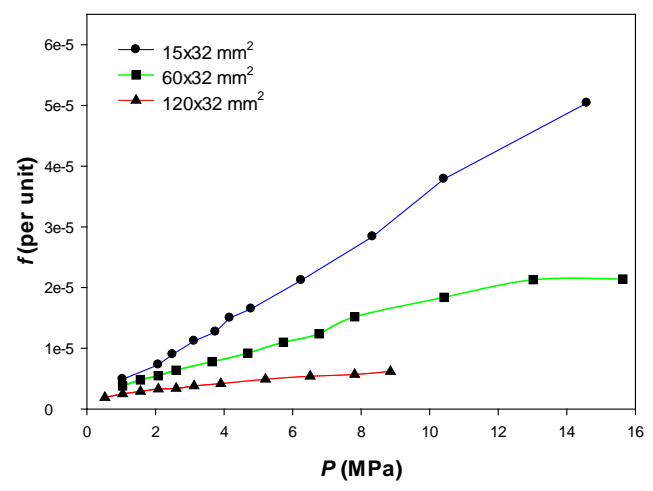

c)

Fig. 7. a) Averaged experimental curves $R_{c, \text { exp }}\left(P, A_{a}\right)$ obtained from 12 measurements each (Step 1 in the flowchart shown in Fig. 5). b) FEM curves $R_{c, F E M}\left(f, A_{a}\right)$ (Step 2 in the flowchart shown in Fig. 5). c) Results of the $f-P$ interpolation between curves in Figs. 7a and 7b (Step 3, see doted lines Figs. 7a and 7b).

Results from Figs. 7a and 7b show that both, the experimental $R_{c, \exp }\left(P, A_{a}\right)$ and the FEM simulated $R_{c, F E M}\left(f, A_{a}\right)$ curves have a similar shape, thus it can facilitate to obtain a relationship between the applied pressure $P$ and the ratio $f=A_{c} / A_{a}$ between the conducting and apparent areas of contact. Experimental results shown in Fig. 7a clearly show that for contact pressures higher than approximately $10 \mathrm{MPa}$, the constriction resistance is almost independent of the apparent area of contact. Fig. 7c shows the results of the $f-P$ interpolation between curves in Figs. $7 \mathrm{a}$ and $7 \mathrm{~b}$.

Table II summarizes the parameters of the fit in (5) corresponding to Step 4, whereas Table III summarizes the parameters of the fit in (6) corresponding to Step 5.

TABLE II

PARAMETERS OF THE LINEAR FIT IN STEP 4

\begin{tabular}{ccccc}
\hline \hline Dimensions $\left(\mathrm{mm}^{2}\right)$ & $A_{a}\left(\mathrm{~mm}^{2}\right)$ & $a\left(\mu \Omega \cdot \mathrm{mm}^{2}\right)$ & $b\left(\mu \Omega \cdot \mathrm{mm}^{2}\right)$ & $R^{2}(-)$ \\
\hline 15x32 & 480 & 0.0084 & 0.0721 & 0.9914 \\
60x32 & 1920 & 0.0084 & 0.0611 & 0.9994 \\
120x32 & 3840 & 0.0084 & 0.1080 & 0.9998 \\
Average $^{1}$ & - & $\mathbf{0 . 0 0 8 4}$ & $\mathbf{0 . 0 8 0 4}$ & - \\
\hline \hline
\end{tabular}

${ }^{1}$ Values applied in equation (5)

TABLE III

PARAMETERS OF THE LINEAR FIT IN STEP 5

\begin{tabular}{ccccc}
\hline \hline Dimensions $\left(\mathrm{mm}^{2}\right)$ & $A_{a}\left(\mathrm{~mm}^{2}\right)$ & $d\left(\mathrm{~mm}^{2} / \mathrm{MPa}\right)$ & $e(-)$ & $R^{2}(-)$ \\
\hline $15 \times 32$ & 480 & 0.0016 & $3.464 \cdot 10^{-7}$ & 0.9974 \\
$60 \times 32$ & 1920 & 0.0025 & $3.137 \cdot 10^{-6}$ & 0.9707 \\
$120 \times 32$ & 3840 & 0.0019 & $2.101 \cdot 10^{-6}$ & 0.9742 \\
Average $^{1}$ & - & $\mathbf{0 . 0 0 2 0}$ & $\mathbf{1 . 8 6 2} \cdot \mathbf{1 0}^{-6}$ & - \\
\hline \hline
\end{tabular}

${ }^{1}$ Values applied in equation (6)

Finally, Figs. 8 summarize the results attained, including the final constriction resistance of the three sets of samples predicted by the proposed approach. 

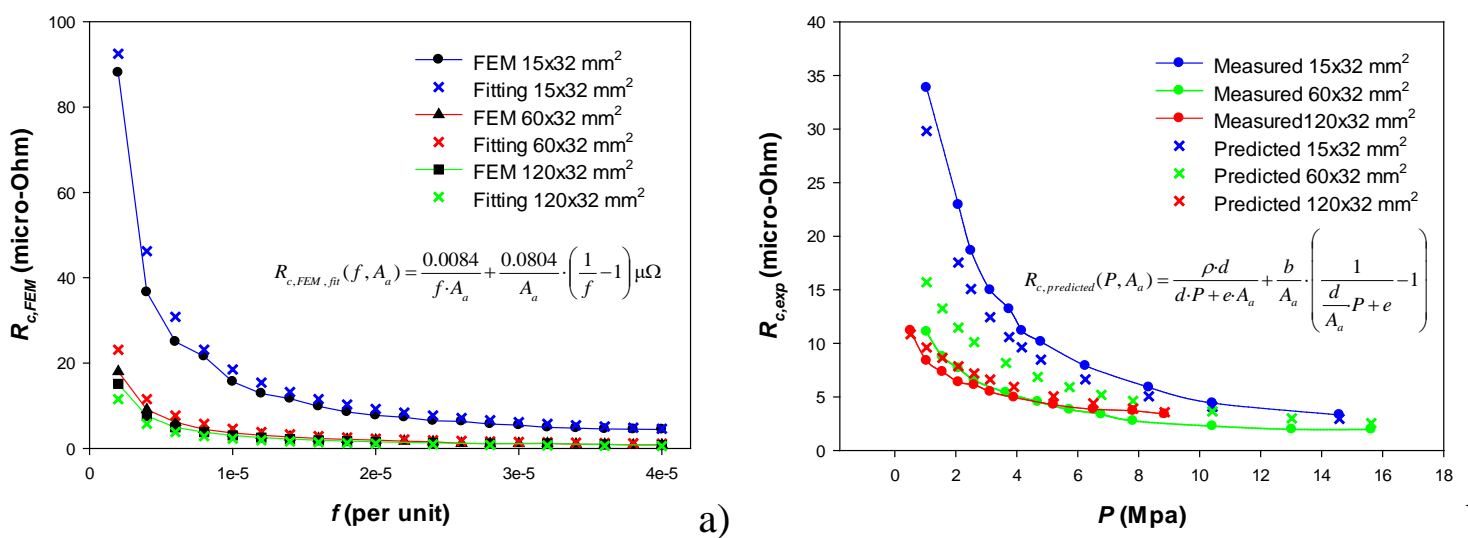

Fig. 8. a) FEM curves $R_{c, F E M}\left(f, A_{a}\right)$ and the fitted values $R_{c, F E M, f i t}\left(f, A_{a}\right)=0.0084 /\left(f \cdot A_{a}\right)+\left(b / A_{a}\right) \cdot(1 / f-1)$ with $b=0.0804$ (Step 4 in the flowchart shown in Fig. 5). b) Averaged experimental curves $R_{c, e x p}\left(P, A_{a}\right)$ and the fitted values from eq. (7) corresponding to Step 6.

It is noted that Fig. 8b shows the constriction resistance of the analyzed copper samples predicted by the method proposed in this paper. The results presented are within the tolerance margin of the experimental measures due to the inherent variability among different samples.

\section{CONCLUSION}

The thermal behavior and expected service life of power connectors and other electrical devices are greatly influenced by the electrical constriction resistance. Therefore it is highly appealing to dispose of predictive models to determine the electrical constriction resistance of devices not yet manufactured, during the design stage. To this end, a simple FEM-based methodology to estimate the electrical constriction resistance of two contacting rough surfaces is proposed. The approach developed in this work requires measurements of the surface roughness and knowledge of the contact pressure, materials and the apparent area of contact. The influence of the contact pressure has also been analyzed in detail. A random array of 3D orthogonal parallelepipeds is generated to account for the stochastic distribution of the spots across the contact interface. The elastic-plastic behavior of the spots is also considered since the number of spots generated in the3D-FEM model is changed and related to the contact pressure according to the dependence $f=f(P)$. It has been shown that the proposed FEM-based model allows predicting both the spots density and the constriction resistance as a function of the pressure. Results from the FEM-based approach show good agreement with experimental measurements of the constriction resistance of the samples analyzed. It has also been proved that when the contact pressure is beyond a certain threshold, the constriction resistance is almost independent of the apparent area of contact. Although this work has analyzed different copper samples with different apparent contact areas, the proposed methodology can be applied to other metallic rough surfaces.

\section{ACKNOWLEDGEMENTS}

This work was partially supported by the Spanish Ministry of Economy and Competitiveness under project 
RTC-2014-2862-3and Generalitat de Catalunya under projects SGR 101 2014-2016 and 2013 DI 024, respectively.

\section{REFERENCES}

[1] F. Capelli, J.-R. Riba, A. Rodriguez, and S. Lalaouna, "Research Towards Energy-Efficient Substation Connectors,” Springer International Publishing, 2017, pp. 295-301.

[2] I. M. G. González-Díaz, D. Pastor, E. García-Hemme, D. Montero, R. García-Hernansanz, J. Olea, A. del Prado, E. San Andrés, “A robust method to determine the contact resistance using the van der Pauw set up,” Measurement, vol. Online, pp. 1-18, 2017.

[3] M. Braunovic, "Effect of connection design on the contact resistance of high power overlapping bolted joints,” IEEE Trans. Components Packag. Technol., vol. 25, no. 4, pp. 642-650, Dec. 2002.

[4] M. Braunovic, N. K. Myshkin, and V. V. Konchits, Electrical Contacts: Fundamentals, Applications and Technology. Boca Raton, FL: CRC Press, Taylor \& Francis Group, 2006.

[5] R. Holm, Electric Contacts - Theory and Application Springer. New York: Springer-Verlag, 1967.

[6] R. Tzeneva, "Investigation of High Power Bolted Busbar Connectors with Longitudinal Slots," Przegląd Elektrotechniczny, pp. 223-226, 2012.

[7] W. E. Wilson, S. V. Angadi, and R. L. Jackson, "Surface separation and contact resistance considering sinusoidal elastic-plastic multi-scale rough surface contact,” Wear, vol. 268, no. 1-2, pp. 190-201, Jan. 2010.

[8] J. Bao, G. Wu, and B. Gao, "Research on electric contact calculation and its heat of 500kV transmission line clamps," in 2016 IEEE International Conference on High Voltage Engineering and Application (ICHVE), 2016, pp. 1-4.

[9] A. J. Robinson, A. M. Lyons, and R. Kempers, "Modelling the effective thermal conductivity of compressing structures including contact resistance,” Simul. Model. Pract. Theory, vol. 67, pp. 7488, 2016.

[10] S. Kucharski and G. Starzynski, "Study of contact of rough surfaces: Modeling and experiment," Wear, vol. 311, no. 1, pp. 167-179, 2014.

[11] R. Femi, A. Agrawal, and S. Clement, “An FEM Study of the Electrothermal Properties of Microelectrical Contacts for Application in the Design of Arcless Switches,” IEEE Trans. Components, Packag. Manuf. Technol., vol. 6, no. 3, pp. 407-417, Mar. 2016.

[12] S. V. Angadi, W. E. Wilson, R. L. Jackson, G. T. Flowers, and B. I. Rickett, “A Multi-Physics Finite Element Model of an Electrical Connector Considering Rough Surface Contact,” in 2008 Proceedings of the 54th IEEE Holm Conference on Electrical Contacts, 2008, pp. 168-177.

[13] J. A. Greenwood and J. B. P. Williamson, “Contact of Nominally Flat Surfaces,” Proc. R. Soc. A Math. Phys. Eng. Sci., vol. 295, no. 1442, pp. 300-319, Dec. 1966.

[14] F. Pennec, D. Peyrou, D. Leray, P. Pons, R. Plana, and F. Courtade, "Impact of the Surface Roughness Description on the Electrical Contact Resistance of Ohmic Switches Under Low Actuation Forces,” IEEE Trans. Components, Packag. Manuf. Technol., vol. 2, no. 1, pp. 85-94, Jan. 2012.

[15] T. Fiedler, N. White, M. Dahari, and K. Hooman, “On the electrical and thermal contact resistance of metal foam,” Int. J. Heat Mass Transf., vol. 72, pp. 565-571, 2014.

[16] H. Liu, D. Leray, P. Pons, and S. Colin, "Finite element multi-physics modeling for ohmic contact of 
microswitches,” in 2014 15th International Conference on Thermal, Mechanical and Mulit-Physics Simulation and Experiments in Microelectronics and Microsystems (EuroSimE), 2014, pp. 1-8.

[17] J. R. Polchow et al., “A Multi-Physics Finite Element Analysis of Round Pin High Power Connectors," in 2010 Proceedings of the 56th IEEE Holm Conference on Electrical Contacts, 2010, pp. 1-9.

[18] P. Shanthraj, O. Rezvanian, and M. A. Zikry, "Electrothermomechanical Finite-Element Modeling of Metal Microcontacts in MEMS,” J. Microelectromechanical Syst., vol. 20, no. 2, pp. 371-382, Apr. 2011.

[19] H. Liu, D. Leray, S. Colin, P. Pons, and A. Broué, "Finite Element Based Surface Roughness Study for Ohmic Contact of Microswitches,” in 2012 IEEE 58th Holm Conference on Electrical Contacts (Holm), 2012, pp. 1-10.

[20] S. V. Angadi, R. L. Jackson, S. Choe, G. T. Flowers, B.-Y. Lee, and L. Zhong, “A Multi-Physics Finite Element Model of a 35A Automotive Connector Including Multiscale Rough Surface Contact,” in 2010 Proceedings of the 56th IEEE Holm Conference on Electrical Contacts, 2010, pp. $1-11$.

[21] Q. Shen, J. Qiu, G. Liu, K. Lv, and Y. Zhang, "Rough surface simulation and electrical contact transient performance," in 2016 Prognostics and System Health Management Conference (PHMChengdu), 2016, pp. 1-6.

[22] H. Liao, Xiaogang; Li, Renxi; Luo, Weifang; Zhang, "Method for measuring thermal contact resistance of graphite thin film materials,” Measurement, vol. 93, no. November 2016, pp. 1-17, 2016.

[23] M. Nakamura and I. Minowa, "Computer Simulation for the Conductance of a Contact Interface," IEEE Trans. Components, Hybrids, Manuf. Technol., vol. 9, no. 2, pp. 150-155, Jun. 1986.

[24] ISO, "ISO 4287:1997 Geometrical Product Specifications (GPS) -- Surface texture: Profile method - Terms, definitions and surface texture parameters.” p. 25, 1997.

[25] A. Laor, P. J. Herrell, and M. Mayer, “A Study on Measuring Contact Resistance of Ball Bonds on Thin Metallization,” IEEE Trans. Components, Packag. Manuf. Technol., vol. 5, no. 5, pp. 704-708, May 2015.

[26] W. Wang et al., "Nanoindentation measurement of hardness and modulus anisotropy in Ni3Al single crystals,” J. Mater. Res., vol. 17, no. 9, pp. 2314-2320, Sep. 2002.

[27] N. K. Myshkin, M. I. Petrokovets, and S. A. Chizhik, "Basic problems in contact characterization at nanolevel,” Tribol. Int., vol. 32, no. 7, pp. 379-385, 1999. 